\title{
Phytoprotection
}

\section{Effet de la dose de semis et de l'écartement entre les rangs sur l'interférence entre le Chenopodium album et le Triticum aestivum}

\author{
D. Pageau et G.F. Tremblay
}

Volume 77, numéro 3, 1996

URI : https://id.erudit.org/iderudit/706108ar

DOI : https://doi.org/10.7202/706108ar

Aller au sommaire du numéro

Éditeur(s)

Société de protection des plantes du Québec (SPPQ)l

ISSN

0031-9511 (imprimé)

1710-1603 (numérique)

Découvrir la revue

Citer cet article

Pageau, D. \& Tremblay, G. (1996). Effet de la dose de semis et de l'écartement entre les rangs sur l'interférence entre le Chenopodium album et le Triticum aestivum. Phytoprotection, 77(3), 119-128. https://doi.org/10.7202/706108ar
Résumé de l'article

Un essai répété 4 années a permis d'évaluer l'effet de la présence du chénopode (Chenopodium album) sur la productivité du blé (Triticum aestivum 'Max'). La céréale a été ensemencée à cinq doses de semis (100, 200,300, 400 et 500 grains viables $\mathrm{m}^{-2}$ ) et à deux écartements entre les rangs $(10$ et $18 \mathrm{~cm})$. Une réduction de l'écartement entre les rangs a permis d'accroître les rendements en blé de $16 \%$ en présence ou non du chénopode. La dose de semis est un facteur plus important que l'écartement entre les rangs. À une dose de semis de 100 grains $\mathrm{m}^{-2}$, la présence du chénopode a causé une réduction du rendement en grain de $23 \%$ tandis que cette réduction était de $11 \%$ avec une dose de semis de 500 grains $\mathrm{m}^{-2}$. La baisse de rendement en blé provoquée par la présence de chénopode est surtout attribuable à une réduction du nombre d'épis par unité de surface. Le chénopode a également réduit le nombre d'épis par plant et le nombre de grains par épi tandis que le poids de 1000 grains et l'indice de récolte ont été peu affectés. Les contenus en azote et en phosphore du grain de blé ont été réduits par la présence du chénopode. Une réduction de l'écartement entre les rangs ou une augmentation de la dose de semis du blé n'a pas permis de réduire le nombre de plants mais a réduit la biomasse du chénopode. 


\title{
Effet de la dose de semis et de l'écartement entre les rangs sur l'interférence entre le Chenopodium album et le Triticum aestivum
}

\author{
Denis Pageau' ${ }^{1}$ et Gaëtan F. Tremblay ${ }^{1}$
}

Reçu 1996-07-09; accepté 1997-01-16

Un essai répété 4 années a permis d'évaluer l'effet de la présence du chénopode (Chenopodium album) sur la productivité du blé (Triticum aestivum 'Max'). La céréale a été ensemencée à cinq doses de semis $(100,200,300,400$ et 500 grains viables $\mathrm{m}^{-2}$ ) et à deux écartements entre les rangs (10 et $18 \mathrm{~cm}$ ). Une réduction de l'écartement entre les rangs a permis d'accroître les rendements en blé de $16 \%$ en présence ou non du chénopode. La dose de semis est un facteur plus important que l'écartement entre les rangs. À une dose de semis de 100 grains $\mathrm{m}^{-2}$, la présence du chénopode a causé une réduction du rendement en grain de $23 \%$ tandis que cette réduction était de $11 \%$ avec une dose de semis de 500 grains $\mathrm{m}^{-2}$. La baisse de rendement en blé provoquée par la présence de chénopode est surtout attribuable à une réduction du nombre d'épis par unité de surface. Le chénopode a également réduit le nombre d'épis par plant et le nombre de grains par épi tandis que le poids de 1000 grains et l'indice de récolte ont été peu affectés. Les contenus en azote et en phosphore du grain de blé ont été réduits par la présence du chénopode. Une réduction de l'écartement entre les rangs ou une augmentation de la dose de semis du blé n'a pas permis de réduire le nombre de plants mais a réduit la biomasse du chénopode.

Pageau, D., and G.F. Tremblay. 1996. Interference between Chenopodium album and Triticum aestivum at different seeding rates and row spacings. PHYTOPROTECTION 77 : 119-128.

A 4-yr study was conducted to evaluate the effect of two row spacings (10 and $18 \mathrm{~cm})$ and five seeding rates $\left(100,200,300,400\right.$, and 500 viable seeds $\left.\mathrm{m}^{-2}\right)$ of wheat (Triticum aestivum 'Max') on the interference between lamb'squarters (Chenopodium album) and the crop. Narrow row spacing increased wheat yield by $16 \%$ regardless of the presence or absence of lamb's-quarters. Seeding rate was a more important factor than row spacing. At a seeding rate of 100 seeds $\mathrm{m}^{-2}$, the yield reduction caused by lamb's-quarters was $23 \%$ whereas it was $11 \%$ when wheat was seeded at 500 seeds $\mathrm{m}^{-2}$. Yield reductions were attributed to a decrease in the number of spikes $\mathrm{m}^{-2}$. Lamb's-quarters also reduced the number of spikes per plant and the number of kernels per spike, and, to a lesser extent, the 1000 kernel weight and the harvest index. Nitrogen and phosphorus content of wheat grain were reduced by the presence of Chenopodium. High seeding rates or narrow row spacing did not reduce the density of lamb's-quarters but did reduce the weed biomass.

1. Agriculture et Agroalimentaire Canada, Ferme de Recherche, Normandin (Québec), Canada G8M 4K3. Contribution n 535 du Centre de recherche et de développement sur les sols et les grandes cultures d'Agriculture et Agroalimentaire Canada, Sainte-Foy (Québec), Canada 


\section{INTRODUCTION}

Le chénopode blanc (Chenopodium album L.) est une mauvaise herbe que I'on rencontre fréquemment dans les provinces et territoires du Canada (Bassett et Crompton 1978). Au Québec, cette plante adventice est présente dans presque tous les champs de céréales (Lemieux et al. 1988).

Des études ont démontré que le chénopode blanc entraîne une réduction des rendements du maïs (Zea mays L.)(Sibuga et Bandeen 1980), du soja [Glycine max (L.) Merr.] (Crook et Renner 1990; Harrison 1990), du tournesol (Helianthus annuus L.)(Szente et al. 1993) et du colza (Brassica napus L.) (Blackshaw et al. 1987). Chez l'avoine (Avena sativa L.), le seuil de nuisibilité du chénopode, c'est-à-dire le niveau de densité au-delà duquel le rendement de la culture diminue significativement, est d'environ 1000 plants $\mathrm{m}^{-2}$ (Lapointe et al. 1984). Chez l'orge (Hordeum vulgare L.), des populations de chénopodes de 150 et 300 plants $\mathrm{m}^{-2}$ ont réduit les rendements en grain de $23 \%$ et $36 \%$ respectivement (Conn et Thomas 1987). Des essais effectués en pots indiquent également que la présence de cette mauvaise herbe réduit les rendements du blé (Triticum aestivum L.) (Bhaskar et Vyas 1988).

Certaines pratiques culturales peuvent réduire l'interférence entre les céréales et les mauvaises herbes. Une augmentation de la dose de semis des céréales réduit les pertes associées à la présence des mauvaises herbes (Barton et al. 1992; Mann et Barnes 1950). Pour une même dose de semis, une réduction de l'écartement entre les rangs permet une distribution plus uniforme des plantes. Des essais effectués avec le blé $d$ 'automne et le brome des seigles (Bromus secalinus L.) ont démontré qu'une réduction de l'écartement entre les rangs permet de réduire les pertes de rendements provoquées par la mauvaise herbe (Koscelny et al. 1990; Solie et al. 1991).

Au Québec, peu d'études ont permis d'évaluer le rôle de l'espacement entre les rangs et de la dose de semis sur l'interférence entre les céréales et les mauvaises herbes. Des travaux antérieurs effectués avec une graminée vivace, le chiendent [Elytrigia repens (L.) Nevski], et l'orge ont démontré qu'une réduction de l'écartement entre les rangs n'a pas permis de réduire l'interférence entre les deux espèces. Cependant, une augmentation de la dose de semis permettait d'accroître les rendements de l'orge en présence de la mauvaise herbe (Pageau et Tremblay 1995).

Cet essai a été entrepris afin de déterminer l'influence d'une réduction de l'écartement entre les rangs et d'une augmentation de la dose de semis sur l'interférence entre une mauvaise herbe annuelle, le chénopode blanc, et le blé de printemps.

\section{MATÉRIEL ET MÉTHODES}

Cette étude a été réalisée de 1988 à 1991 à Normandin (lat. 48 $51^{\circ} \mathrm{N}$, long. $72^{\circ} 32^{\prime} \mathrm{O}$ ) (Québec) sur un gleysol humique série Normandin. Les effets de cinq doses de semis du cultivar de blé 'Max' $(100,200$, 300,400 et 500 grains viables $\mathrm{m}^{-2}$ ), de deux écartements entre les rangs (10 et $18 \mathrm{~cm}$ ) et de deux niveaux d'infestation de chénopode (présence ou absence) ont été évalués. Cet essai a été répété à chaque année dans une nouvelle portion $d^{\prime}$ un champ où la population naturelle de chénopodes était très élevée. Un traitement témoin de chénopodes en absence de céréale était également inclus. Pour ce traitement, les autres mauvaises herbes, principalement la stellaire moyenne [Stellaria media (L.) Cyrill.] et I'ortie royale (Galeopsis tetrahit L.), étaient enlevées manuellement. Pour les parcelles où le chénopode devait être absent, une application de $281 \mathrm{~g} \mathrm{~m} . \mathrm{a}$. ha-1 de cyanazine [2-[[4-chloro-6-(éthylamino)-1,3,5-triazine2-yl]amino]-2-méthylpropanenitrile)] et de $563 \mathrm{~g} \mathrm{~m}$.a. ha-1 de MCPA [acide (4-chloro2-méthylphénoxy) acétique] en mélange, a été effectuée au stade 4-5 feuilles de la céréale. Les mauvaises herbes qui échappaient au traitement étaient enlevées manuellement.

Avant le semis, des applications de $80 \mathrm{~kg} \mathrm{ha}^{-1} \mathrm{~d}^{\prime}$ azote et de $30 \mathrm{~kg} \mathrm{ha}^{-1}$ de $\mathrm{P}_{2} \mathrm{O}_{5}$ et $\mathrm{K}_{2} \mathrm{O}$ ont été effectuées. Par la suite, une application de $60 \mathrm{~kg} \mathrm{ha}^{-1} \mathrm{~d}^{\prime}$ azote a été effectuée au stade début tallage de la céréale. Chaque parcelle était constituée de huit rangs (écartement de $18 \mathrm{~cm}$ ) ou 
de 16 rangs (écartement $10 \mathrm{~cm}$ ) d'une longueur de $6 \mathrm{~m}$.

Environ 2 semaines après l'émergence, les plantules de céréales ont été comptées sur deux sections d'une longueur de $1 \mathrm{~m}$ déterminées au hasard parmi les rangs du centre de chaque parcelle. Le nombre d'épis a également été évalué sur ces mêmes sections et le nombre d'épis par plant a été déterminé en divisant le nombre d'épis par le nombre de plantules. Ces sections ont été récoltées séparément du reste de la parcelle. Le poids total (poids de la paille et du grain) a été déterminé et le grain a été battu, pesé et compté. L'indice de récolte a été obtenu en divisant le poids du grain par le poids total. Le nombre moyen de grains produit par épi a été évalué en divisant le nombre de grains total par le nombre d'épis. Quelques jours avant la récolte des céréales, les plants de chénopode ont été comptés à l'intérieur de deux quadrats de $0,09 \mathrm{~m}^{2}(30 \mathrm{~cm} \times 30 \mathrm{~cm})$ sélectionnés au hasard dans chacune des parcelles. Ces plants ont été récoltés, séchés à $105^{\circ} \mathrm{C}$ pendant $48 \mathrm{~h}$ et pesés.

Le rendement en grain a été déterminé en récoltant les quatre rangs (écartement de $18 \mathrm{~cm}$ ) ou huit rangs (écartement de $10 \mathrm{~cm}$ ) du centre de chaque parcelle sur une longueur de $5,5 \mathrm{~m}$. Le poids de 1000 grains a été obtenu en comptant et en pesant un échantillon de 1000 grains.

Un échantillon de grain représentatif de la récolte de chaque parcelle a été prélevé, séché à $45^{\circ} \mathrm{C}$ et moulu en utilisant un tamis de $1 \mathrm{~mm}$ sur une moulange Wiley (Arthur H. Thomas Co., Philadelphie, PA). Les échantillons ont été minéralisés en utilisant un mélange d'acide sulfurique et d'acide sélénieux tel que décrit dans la méthode d'Isaac et Johnson (1976). L'azote total a été déterminé sur un autoanalyseur Lachat (Lachat Instruments, Milwaukee, WI) en utilisant la méthode 13-107-06-2-D (Lachat Instruments 1992). Le phosphore dans les solutions issues de la minéralisation a été mesuré en utilisant la méthode décrite par le CPVQ (1988) alors que le potassium, le calcium et le magnésium ont été analysés par absorption atomique (Richards 1993). Du lanthane était ajouté aux solutions issues de la minéralisation pour une concentration finale de 0,22 \% (poids:vol.) avant le dosage du $\mathrm{Ca}$ et du Mg. La matière sèche des échantillons a été déterminée après séchage à l'étuve à $105^{\circ} \mathrm{C}$ pendant $24 \mathrm{~h}$. La composition chimique des échantillons a été exprimée sur une base de matière sèche.

Le dispositif expérimental utilisé était un plan en bloc complet avec arrangement factoriel des traitements répétés quatre fois (deux écartements, deux niveaux de chénopodes et cinq doses de semis). L'analyse de variance a été effectuée en utilisant la procédure GLM de SAS (SAS Institute 1990).

\section{RÉSULTATS ET DISCUSSION}

Les résultats de l'analyse de variance sur les différents paramètres mesurés sur le blé sont présentés au Tableau 1. Peu importe l'année, la dose de semis, la présence ou l'absence de chénopodes, une réduction de l'écartement entre les rangs a permis d'accroître les rendements de la céréale. En moyenne, le rendement en grain du blé était $16 \%$ supérieur (1206 $\mathrm{kg} \mathrm{ha}^{-1}$ ) avec un écartement entre les rangs de $10 \mathrm{~cm}$ comparativement à un écartement de $18 \mathrm{~cm}$ (1039 $\left.\mathrm{kg} \mathrm{ha}^{-1}\right)$. Comme dans les essais de Koscelny et al. $(1990,1991)$ et ceux de Solie et al. (1991), une réduction de l'écartement entre les rangs a permis d'accroître les rendements en grain lorsque la céréale était en présence ou non de la mauvaise herbe.

La présence de chénopodes a réduit le nombre de grains produits par épi de blé, peu importe l'écartement entre les rangs utilisé (Tableau 1). En absence de chénopode, les épis ont produit en moyenne 28 grains tandis qu'il n'y avait que 25 grains par épi lorsque la mauvaise herbe était présente. Bhaskar et Vyas (1988) ont indiqué également que la présence du chénopode réduisait le nombre de grains par épi de blé. En accord avec les résultats de Cussans et Wilson (1975) et ceux de Pageau et Tremblay (1995), la dose de semis est un facteur qui influence la productivité de la céréale de façon plus importante qu'une variation de l'écartement entre les rangs, que la céréale soit en présence ou non de mauvaises herbes (Tableau 1). 
Tableau 1. Analyse de variance du rendement, des facteurs associés au rendement et de la composition du grain de blé

\begin{tabular}{|c|c|c|c|c|c|c|c|c|c|}
\hline \multirow[b]{2}{*}{ Source } & \multirow{2}{*}{$\begin{array}{l}\text { Degré } \\
\text { de } \\
\text { liberté }\end{array}$} & \multirow[b]{2}{*}{ Rendement } & \multirow{2}{*}{$\begin{array}{l}\text { Poids de } \\
1000 \text { grains }\end{array}$} & \multirow{2}{*}{$\begin{array}{c}\text { Nombre } \\
\text { d'épis } \\
\text { plant }^{-1}\end{array}$} & \multirow{2}{*}{$\begin{array}{l}\text { Nombre de } \\
\text { grains épi }{ }^{-1}\end{array}$} & \multirow{2}{*}{$\begin{array}{l}\text { Densité } \\
\text { des épis }\end{array}$} & \multirow{2}{*}{$\begin{array}{l}\text { Indice de } \\
\text { récolte }\end{array}$} & \multicolumn{2}{|c|}{$\begin{array}{c}\text { Composition } \\
\text { du grain }\end{array}$} \\
\hline & & & & & & & & $N$ & $P$ \\
\hline Année (A) & 3 & $* *$ & $* *$ & $* *$ & $* *$ & $* *$ & $* *$ & $* *$ & $* *$ \\
\hline Écartement $(E)$ & 1 & $* *$ & NS & NS & NS & $* *$ & NS & NS & NS \\
\hline Dose (D) & 4 & $* *$ & $* *$ & $* *$ & $* *$ & $* *$ & $* *$ & NS & $* *$ \\
\hline Chénopode $(\mathrm{C})$ & 1 & $* *$ & $* *$ & ** & $* *$ & $* *$ & $* *$ & $* *$ & ** \\
\hline$E \times A$ & 3 & NS & NS & * & NS & NS & $* *$ & NS & NS \\
\hline$D \times A$ & 4 & $* *$ & $* *$ & $* *$ & $* *$ & $* *$ & NS & NS & NS \\
\hline$C \times A$ & 3 & $* *$ & $* *$ & $* *$ & $* *$ & $* *$ & $* *$ & NS & NS \\
\hline$E \times D$ & 4 & NS & NS & NS & NS & NS & NS & NS & NS \\
\hline$E X C$ & 1 & NS & NS & NS & $*$ & NS & NS & NS & NS \\
\hline$D \times C$ & 4 & $* *$ & NS & $* *$ & ** & NS & NS & $* *$ & NS \\
\hline$D \times C \times A^{a}$ & 12 & NS & NS & $* *$ & $* *$ & $* *$ & NS & NS & NS \\
\hline
\end{tabular}

${ }^{*},{ }^{* *}$ significatif à $P \leq 0,05$ et 0,01 , respectivement; NS, non significatif.

a Les autres interactions triples ainsi que l'interaction EXDXCXA n'étaient pas significatives pour les paramètres mesurés.

Le chénopode blanc a réduit les rendements en grain du blé (Fig. 1). Les essais de Conn et Thomas (1987) avec l'orge et ceux de Welbank (1963), de Shahi (1978) et de Bhaskar et Vyas (1988) avec le blé ont indiqué également que cette mauvaise herbe réduit le rendement en grain des céréales. À une dose de semis de 100 grains $\mathrm{m}^{-2}$, le chénopode a causé une réduction du rendement en grain de $23 \%$ tandis que cette réduction était de $11 \%$ avec une dose de semis de 500 grains $\mathrm{m}^{-2}$. Une augmentation de la dose de semis de la céréale a donc permis de limiter l'effet négatif du chénopode. En accord avec Erviö (1972), le rendement du blé est réduit par les mauvaises herbes mais une augmentation de la dose de semis a permis d'accroître les rendements. En absence de chénopodes, une augmentation de la dose de semis de 100 à 500 grains $\mathrm{m}^{-2}$ a permis d'augmenter le rendement en grain de $45 \%$ en moyenne, alors qu'en présence de la mauvaise herbe cette augmentation était de $100 \%$ (Fig. 1).

En 1988, 1990 et 1991, comparativement à une dose de semis de 500 grains $\mathrm{m}^{-2}$, l'effet du chénopode sur le nombre d'épis était plus important lorsque le blé était ensemencé à de faibles doses de semis (Tableau 2). Par exemple, en 1988, la présence de chénopodes a causé une réduction de 1,5 épis plant ${ }^{-1}$ lorsque le taux de semis était de 100 grains $\mathrm{m}^{-2}$, alors que cette réduction n'était plus que de 0,5 épi par plant à une dose de semis de 500 grains $\mathrm{m}^{-2}$. En 1989, l'interaction dose de semis $X$ chénopode n'était pas significative mais l'effet de la présence du chénopode sur le nombre d'épis produits par plant était significatif. Morishita et Thill (1988) ainsi que Bhaskar et Vyas (1988) ont aussi démontré que les mauvaises herbes réduisaient le nombre d'épis produits par plant.

En moyenne, le chénopode a récluit de $28,10,38$ et $7 \%$ le nombre d'épis de blé par unité de surface en 1988, 1989, 1990 et 1991, respectivement (Tableau 2). Cependant, en 1988, la réduction du nombre d'épis causée par le chénopode a été de $8 \%$ à une dose de semis de 100 grains $\mathrm{m}^{-2}$ et de $27 \%$ lorsque le blé était ensemencé à une dose de semis de 500 grains $\mathrm{m}^{-2}$. En 1991, le chénopode a réduit la densité des épis de blé sauf pour la dose de semis de 500 grains $\mathrm{m}^{-2}$ où la densité des épis a été plus élevée en présence de la mauvaise herbe. II est cependant difficile d'expliquer cet effet. Gill et al. (1987) ainsi que Mclennan et al. (1991) ont également démontré que la présence de mauvaises herbes provoquait une réduction de la densité des épis de blé. Les résultats de cet essai de même que ceux de Peterson et Nalewaja (1992) avec la sétaire verte [Setaria viridis (L.) Beauv.] et le blé, indiquent que la diminution du rendement en blé est sur- 
Tableau 2. Effet de la dose de semis et de la présence du chénopode sur le nombre d'épis par plant, la densité des épis et le nombre de grains par épi de blé

\begin{tabular}{|c|c|c|c|c|c|c|c|c|c|c|c|c|c|}
\hline \multirow{2}{*}{$\begin{array}{l}\text { Dose de } \\
\text { semis } \\
\text { (grains } \mathrm{m}^{-2} \text { ) } \\
100\end{array}$} & \multirow{2}{*}{$\begin{array}{l}\text { Chénopode } \\
\text { Absence } \\
\text { Présence }\end{array}$} & \multicolumn{4}{|c|}{$\frac{\text { Nombre d'épis plant }{ }^{-1}}{1988198919901991}$} & \multicolumn{4}{|c|}{$\begin{array}{c}\text { Densité des épis } \\
\text { (épis } \mathrm{m}^{-2} \text { ) }\end{array}$} & \multicolumn{4}{|c|}{ Nombre de grains épi ${ }^{-1}$} \\
\hline & & $\begin{array}{l}4,7 \\
3,2\end{array}$ & $\begin{array}{l}3,4 \\
2,7\end{array}$ & $\begin{array}{l}4,9 \\
2,1\end{array}$ & $\begin{array}{l}2,3 \\
1,7\end{array}$ & $\begin{array}{l}271 \\
250\end{array}$ & $\begin{array}{l}230 \\
205\end{array}$ & $\begin{array}{l}307 \\
131\end{array}$ & $\begin{array}{l}189 \\
146\end{array}$ & $\begin{array}{l}30 \\
20\end{array}$ & $\begin{array}{l}35 \\
34\end{array}$ & $\begin{array}{l}34 \\
16\end{array}$ & $\begin{array}{l}32 \\
31\end{array}$ \\
\hline 200 & $\begin{array}{l}\text { Absence } \\
\text { Présence }\end{array}$ & $\begin{array}{l}3,1 \\
2,5\end{array}$ & $\begin{array}{l}2,2 \\
1,9\end{array}$ & $\begin{array}{l}3,0 \\
1,9\end{array}$ & $\begin{array}{l}1,2 \\
1,2\end{array}$ & $\begin{array}{l}388 \\
316\end{array}$ & $\begin{array}{l}302 \\
262\end{array}$ & $\begin{array}{l}429 \\
263\end{array}$ & $\begin{array}{l}207 \\
183\end{array}$ & $\begin{array}{l}25 \\
21\end{array}$ & $\begin{array}{l}32 \\
35\end{array}$ & $\begin{array}{l}30 \\
21\end{array}$ & $\begin{array}{l}30 \\
30\end{array}$ \\
\hline 300 & $\begin{array}{l}\text { Absence } \\
\text { Présence }\end{array}$ & $\begin{array}{l}2,8 \\
2,1\end{array}$ & $\begin{array}{l}1,7 \\
1,5\end{array}$ & $\begin{array}{l}2,1 \\
1,4\end{array}$ & $\begin{array}{l}1,1 \\
1,0\end{array}$ & $\begin{array}{l}426 \\
357\end{array}$ & $\begin{array}{l}330 \\
308\end{array}$ & $\begin{array}{l}436 \\
277\end{array}$ & $\begin{array}{l}268 \\
246\end{array}$ & $\begin{array}{l}24 \\
19\end{array}$ & $\begin{array}{l}33 \\
33\end{array}$ & $\begin{array}{l}28 \\
21\end{array}$ & $\begin{array}{l}28 \\
27\end{array}$ \\
\hline 400 & $\begin{array}{l}\text { Absence } \\
\text { Présence }\end{array}$ & $\begin{array}{l}2,4 \\
1,5\end{array}$ & $\begin{array}{l}1,4 \\
1,3\end{array}$ & $\begin{array}{l}2,0 \\
1,2\end{array}$ & $\begin{array}{l}1,0 \\
0,9\end{array}$ & $\begin{array}{l}530 \\
350\end{array}$ & $\begin{array}{l}357 \\
321\end{array}$ & $\begin{array}{l}512 \\
344\end{array}$ & $\begin{array}{l}336 \\
294\end{array}$ & $\begin{array}{l}22 \\
20\end{array}$ & $\begin{array}{l}32 \\
33\end{array}$ & $\begin{array}{l}27 \\
22\end{array}$ & $\begin{array}{l}25 \\
24\end{array}$ \\
\hline 500 & $\begin{array}{l}\text { Absence } \\
\text { Présence }\end{array}$ & $\begin{array}{l}1,8 \\
1,3\end{array}$ & $\begin{array}{l}1,2 \\
1,1\end{array}$ & $\begin{array}{l}1,6 \\
1,2\end{array}$ & $\begin{array}{l}0,9 \\
0,9\end{array}$ & $\begin{array}{l}558 \\
407\end{array}$ & $\begin{array}{l}392 \\
354\end{array}$ & $\begin{array}{l}508 \\
344\end{array}$ & $\begin{array}{l}309 \\
357\end{array}$ & $\begin{array}{l}22 \\
20\end{array}$ & $\begin{array}{l}31 \\
31\end{array}$ & $\begin{array}{l}25 \\
22\end{array}$ & $\begin{array}{l}24 \\
21\end{array}$ \\
\hline Moyenne & $\begin{array}{l}\text { Absence } \\
\text { Présence }\end{array}$ & $\begin{array}{l}3,0 \\
2,1\end{array}$ & $\begin{array}{l}2,0 \\
1,7\end{array}$ & $\begin{array}{l}2,7 \\
1,6\end{array}$ & $\begin{array}{l}1,3 \\
1,1\end{array}$ & $\begin{array}{l}435 \\
336\end{array}$ & $\begin{array}{l}322 \\
290\end{array}$ & $\begin{array}{l}438 \\
272\end{array}$ & $\begin{array}{l}262 \\
245\end{array}$ & $\begin{array}{l}25 \\
20\end{array}$ & $\begin{array}{l}33 \\
33\end{array}$ & $\begin{array}{l}29 \\
20\end{array}$ & $\begin{array}{l}28 \\
27\end{array}$ \\
\hline Erreur-type & & 0,05 & 0,04 & 0,05 & 0,02 & 6 & 4 & 5 & 4 & 0,3 & 0,3 & 0,4 & 0,3 \\
\hline \multicolumn{14}{|c|}{ Source de variation } \\
\hline \multirow{3}{*}{\multicolumn{2}{|c|}{$\begin{array}{l}\text { Dose de semis } \\
\text { Chénopode }(C) \\
D \times C\end{array}$}} & $* *$ & ** & $* *$ & ** & $* *$ & $* *$ & $* *$ & ** & $* *$ & $* *$ & NS & $* *$ \\
\hline & & ** & ** & ** & ** & ** & ** & $* *$ & * & ** & NS & ** & NS \\
\hline & & * & NS & $* *$ & ** & ** & NS & NS & $* *$ & $* *$ & NS & $* *$ & NS \\
\hline
\end{tabular}

${ }^{*},{ }^{*}$ significatif à $P \leq 0,05$ et 0,01 , respectivement; NS, non significatif.

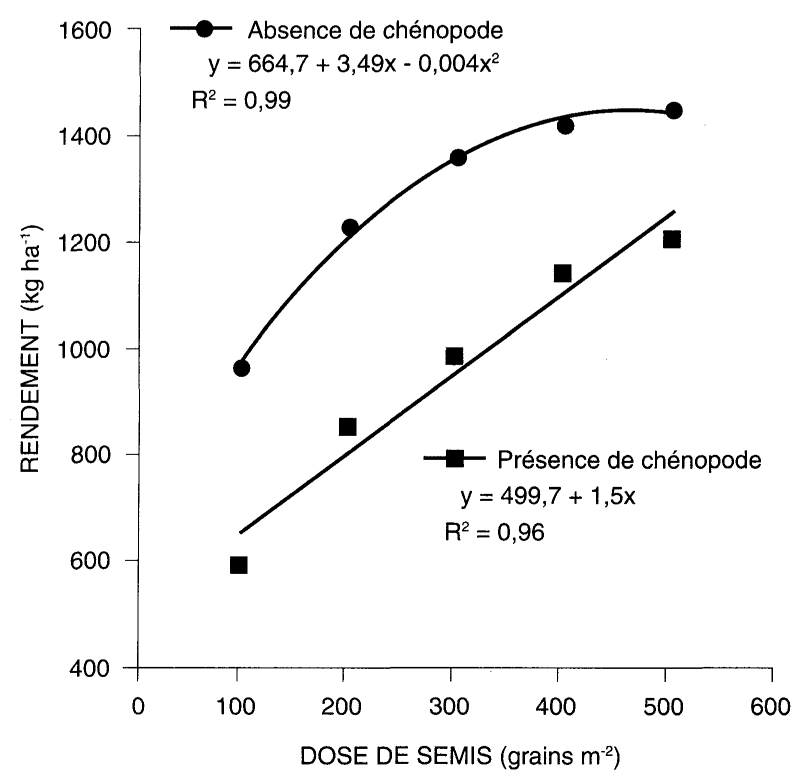

Figure 1. Effet de la dose de semis et de la présence du chénopode sur le rendement en grain du blé. Chaque point représente la moyenne de deux écartements, quatre répétitions et quatre années. 
tout attribuable à une réduction de la densité des épis.

Le chénopode a entraîné une réduction du nombre de grains par épi en 1988 et 1990 seulement (Tableau 2). Cette réduction était importante lorsque la dose de semis était faible. Pour une dose de semis de 100 grains $\mathrm{m}^{-2}$, la présence du chénopode a causé une réduction du nombre de grains par épi de 34 et $64 \%$ en 1988 et 1990 respectivement. Pour une dose de semis de 500 grains $\mathrm{m}^{-2}$, cette réduction n'était plus que de 9 et $12 \%$. Les essais de Bhaskar et Vyas (1988) indiquent également que le chénopode réduit le nombre de grains par épi de blé.

Le poids de 1000 grains a été réduit par la présence du chénopode en 1988, 1989 et 1991 et l'indice de récolte a été réduit en 1988 et 1990 seulement (Tableau 3). Dans leurs essais, Peterson et Nalewaja (1992) ont observé que le poids des grains de blé n'est pas affecté par la présence de sétaire verte.

La compétition pour les éléments nutritifs peut également jouer un rôle important lorsque le blé est en présence de mauvaises herbes. Pour des doses de semis de 100 à 300 grains $\mathrm{m}^{-2}$, la présence du chénopode a peu modifié le contenu en azote du grain de blé (Tableau 4). Pour des doses de semis plus élevées, la présence de la mauvaise herbe a réduit de façon plus importante le contenu en azote. II semble donc que la fertilisation appliquée était suffisante pour répondre aux besoins de la mauvaise herbe et de la céréale lorsque celle-ci était ensemencée à une dose de semis de 100 à 300 grains $\mathrm{m}^{-2}$ mais ne pouvait plus répondre aux besoins des deux espèces pour des doses de 400 et 500 grains $\mathrm{m}^{-2}$. Le contenu en phosphore du grain a été réduit de $4 \%$ lorsque le blé était en présence du chénopode tandis que les contenus en potassium, calcium et magnésium n'ont pas été influencés (données non présentées). Dans cet essai, la réduction de la concentration en $\mathrm{N}$ et $\mathrm{P}$ du grain de blé est significative mais peu élevée. La quantité d'azote appliquée (140 $\left.\mathrm{kg} \mathrm{ha}^{-1}\right)$ était relativement importante et les coritenus en potassium et phosphore du site d'essai étaient également très élevés.

Les travaux de Kapoor et Ramakrishnan (1978) indiquent que les contenus en azote, en phosphore et en potassium du blé sont réduits par la présence du chénopode. Le chénopode est une plante qui consomme beaucoup d'éléments nutritifs, plus particulièrement l'azote, le potassium, le calcium, le magnésium et le fer (Erviö 1971; Shahi 1978; Vengris et al. 1955). D'après Malicki et Berbeciowa (1986), les contenus en N, P, K, Ca, Mg et $\mathrm{Na}$ sont plus élevés dans le chénopode que dans le blé, ce qui en fait un fort compétiteur pour les éléments nutritifs. En accord avec les travaux de Bhaskar et

Tableau 3. Effet de la présence du chénopode sur le poids de 1000 grains et l'indice de récolte du blé

\begin{tabular}{llll}
\hline Année & Chénopode & $\begin{array}{c}\text { Poids de } 1000 \text { grains } \\
(\mathrm{g})\end{array}$ & Indice de récolte \\
\hline 1988 & Absence & 27,2 & 0,37 \\
& Présence & 25,1 & 0,24 \\
& LSD (0,05) & 0,5 & 0,02 \\
1989 & Absence & 38,1 & 0,48 \\
& Présence & 38,6 & 0,48 \\
& LSD (0,05) & 0,3 & NS \\
& Absence & 28,7 & 0,33 \\
& Présence & 28,1 & 0,28 \\
& LSD $(0,05)$ & 0,6 & 0,02 \\
& Absence & 35,3 & 0,48 \\
& Présence & 34,6 & 0,48 \\
& LSD $(0,05)$ & 0,3 & NS \\
\hline
\end{tabular}

NS: non significatif. 
Tableau 4. Effet de la dose de semis et de la présence du chénopode sur le contenu en azote du grain de blé

\begin{tabular}{llr}
\hline $\begin{array}{l}\text { Dose de semis } \\
\left(\text { grains } \mathrm{m}^{-2}\right)\end{array}$ & Chénopode & $\begin{array}{c}\text { Azote } \\
\left(\mathrm{mg} \mathrm{g}^{-1}\right)\end{array}$ \\
\hline 100 & Absence & 3,10 \\
& Présence & 3,00 \\
200 & Absence & 3,05 \\
& Présence & 3,02 \\
300 & Absence & 3,10 \\
& Présence & 3,06 \\
400 & Absence & 3,11 \\
& Présence & 2,92 \\
500 & Absence & 3,12 \\
& Présence & 2,92 \\
Moyenne & Absence & 3,09 \\
& Présence & 2,98 \\
Erreur-type & & 0,01 \\
\hline
\end{tabular}

Vyas (1988), il semble donc que la compétition entre le chénopode blanc et le blé s'effectue surtout pour l'azote et le phosphore.

La présence de la céréale a permis une réduction de $31 \%$ du nombre de plants de chénopodes (Tableau 5). Cependant, une réduction de l'écartement entre les rangs ou une augmentation de la dose de semis de la céréale n'a pas influencé le nombre de plants de la mauvaise herbe.

La biomasse du chénopode a également été réduite par la présence de la céréale (Tableau 5). Comparativement au témoin, la moyenne des doses de semis indique que la présence de la céréale a causé une réduction de $57 \%$ de la biomasse de la mauvaise herbe. Les travaux de Kapoor et Ramakrishnan (1978) indiquaient également que le blé réduit la biomasse du chénopode.

De plus, une réduction de l'écartement entre les rangs a permis de réduire la biomasse de la mauvaise herbe. Un écartement entre les rangs de $10 \mathrm{~cm}$ a permis une réduction de $9 \%$ du poids du chénopode comparativement à un écartement de $18 \mathrm{~cm}$. Une augmentation de la dose de semis de 100 à 500 grains $\mathrm{m}^{-2}$ a réduit la biomasse du chénopode de 58 et $52 \%$ lorsque l'écartement entre les rangs était de 10 et $18 \mathrm{~cm}$ respectivement
(Tableau 5). D'après Erviö (1972), une augmentation de la dose de semis du blé entraîne une réduction du nombre de mauvaises herbes ainsi que du poids individuel et du rendement du chénopode. En accord avec les travaux de Erviö (1972), I'effet d'une augmentation du taux de semis du blé permet de réduire davantage la biomasse des mauvaises herbes plutôt que leur nombre.

Le blé n'a pas permis d'obtenir un contrôle total du chénopode et la mauvaise herbe a pu atteindre sa maturité et ainsi produire des graines. $\mathrm{D}^{\prime}$ après $\mathrm{Ka}$ poor et Ramakrishnan (1978), en semis pur (400 plants $\mathrm{m}^{-2}$ ), le chénopode produit environ 700 graines par plant. Lorsque le blé ( 100 plants $\mathrm{m}^{-2}$ ) est en présence de 300 plants de chénopodes $\mathrm{m}^{-2}$, chaque mauvaise herbe ne produit plus qu'environ 100 graines. Cependant, selon les études, le nombre de graines produites par plant de chénopode varie beaucoup. D'après Erviö (1971), le chénopode peut produire de 250 à 42000 graines par plant selon les années et la densité de la mauvaise herbe. II existe cependant une corrélation positive entre le poids sec de la mauvaise herbe et le nombre de graines produites (Crook et Renner 1990; Harrison 1990). Ainsi, pour chaque gramme de biomasse totale, le chénopode peut produire 1000 graines (Crook et Renner 1990). 
Tableau 5. Effet de l'écartement entre les rangs et de la dose de semis du blé sur la densité et le poids du chénopode ${ }^{a}$

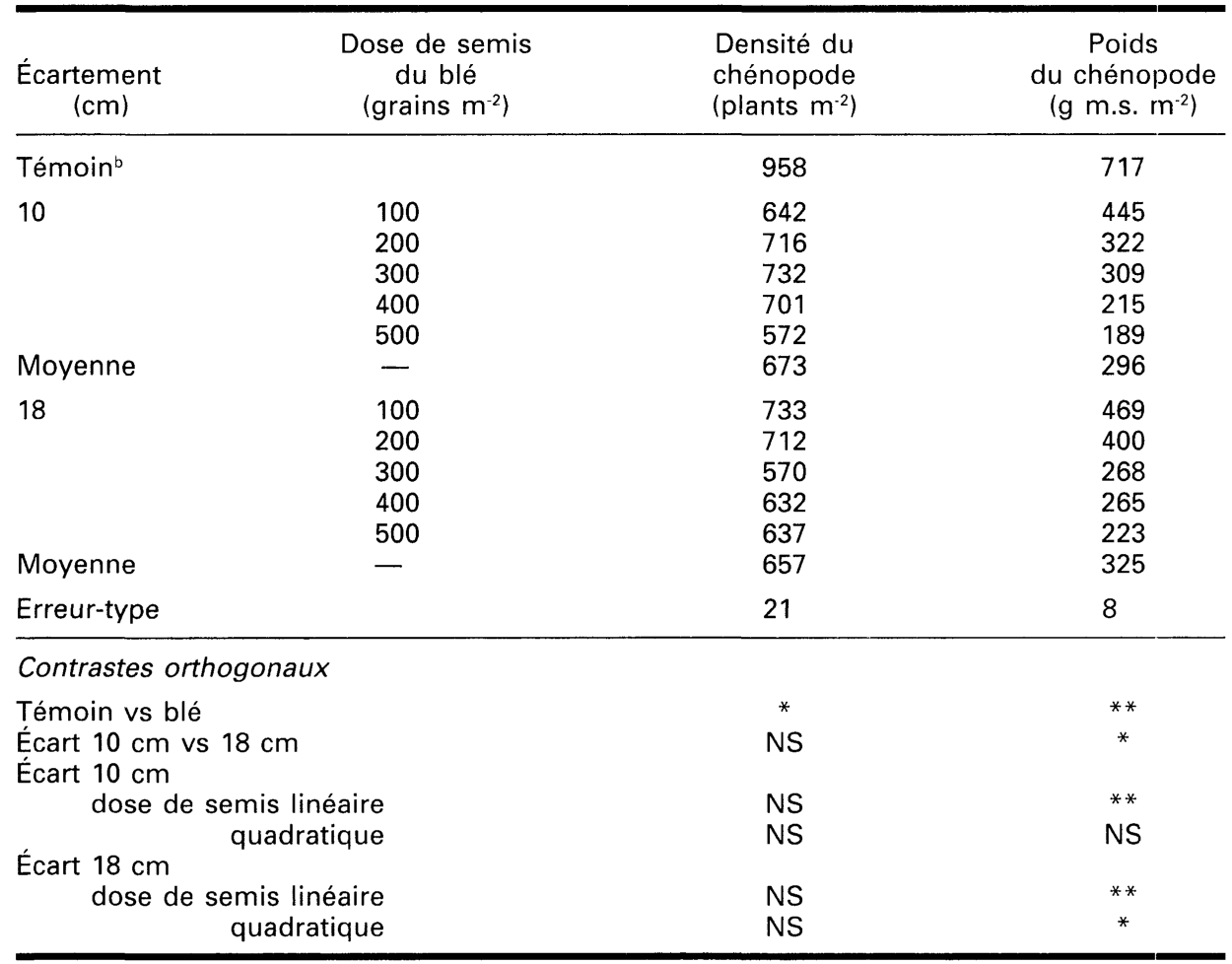

*,** significatif à $P \leq 0,05$ et 0,01 , respectivement; NS, non significatif.

a Moyenne 1988-1991.

b Le traitement témoin excluait la présence de blé.

La diminution de la densité des épis de blé en présence du chénopode pourrait s'expliquer par une forte compétition pour la lumière. Les travaux de Willey et Holliday (1971) indiquent qu'une réduction de l'intensité lumineuse de l'émergence jusqu'à peu de temps après l'initiation des épis de blé entraîne une réduction du rendement en grain du blé attribuable surtout à une réduction de la densité des épis.

Le blé réduit également la lumière disponible pour le chénopode. Les cultivars de blé qui permettent un bon contrôle des mauvaises herbes sont associés à une taille et des rendements en grain élevés (Wicks et al. 1986).

En période de sécheresse, la compétition pour l'eau pourrait également être un facteur à considérer. D'après Shahi (1978), le chénopode est une plante qui utilise beaucoup d'eau. Cette mauvaise herbe puise l'eau uniformément jusqu'à une profondeur de $90 \mathrm{~cm}$. Contrairement au chénopode, le blé puise surtout l'eau dans les premiers $15 \mathrm{~cm}$ de sol. Le système radiculaire du chénopode se développe dans les couches profondes du sol tandis que celui du blé est concentré dans la couche de $0-15 \mathrm{~cm}$. Au cours de notre essai, les précipitations ont été normales et il est peu probable que le blé et le chénopode aient souffert d'un manque d'eau. Durant la saison de croissance (mai-septembre) de 1988, 1989, 1990 et 1991, les précipitations observées au site d'essai ont été de $440,453,443$ et $310 \mathrm{~mm}$, respectivement.

D'après les résultats de cet essai, une réduction de l'écartement entre les rangs ne peut être utilisée pour réduire l'interférence entre le chénopode et le blé. 
Cependant, en présence du chénopode blanc, la productivité du blé est accrue lorsque la dose de semis est élevée. Une réduction de l'écartement entre les rangs et une augmentation de la dose de semis du blé a réduit le nombre et le poids du chénopode. Néanmoins, ces pratiques culturales ne parviennent pas à contrôler efficacement la population de chénopode blanc. De plus, une augmentation des coûts de production associée à une dose de semis élevée du blé ne permettrait probablement pas d'accroître le revenu net des producteurs.

\section{REMERCIEMENTS}

Les auteurs remercient MM. Normand Dallaire et Maurice Roy pour leur aide technique.

\section{RÉFÉRENCES}

Barton, D.L., D.C. Thill et B. Shafii. 1992. Integrated wild oat (Avena fatua) management affects spring barley (Hordeum vulgare) yield and economics. Weed Technol. 6: 129-135.

Bassett, I.J. et C.W. Crompton. 1978. The biology of Canadian weeds. 32. Chenopodium album L. Can. J. Plant Sci. 58: 10611072.

Bhaskar, A. et K.G. Vyas. 1988. Studies on competition between wheat and Chenopodium album L. Weed Res. 28: 53-58.

Blackshaw, R.E., G.W. Anderson et J. Dekker. 1987. Interference of Sinapsis arvensis $\mathrm{L}$. and Chenopodium album L. in spring rapeseed (Brassica napus L.). Weed Res. 27: 207-213.

Conn, J.S. et D.L. Thomas. 1987. Common lambsquarters (Chenopodium album) interference in spring barley. Weed Technol. 1: 312-313.

CPVQ. 1988. Méthodes d'analyse des sols, des fumiers et des tissus végétaux. AGDEX 533. Conseil des productions végétales du Québec, Québec.

Crook, T.M. et K.A. Renner. 1990. Common lambsquarter (Chenopodium album) competition and time of removal in soybeans (Glycine max). Weed Res. 38: 358-364.

Cussans, G.W. et B.J. Wilson. 1975. Some effects of crop row width and seedrate on competition between spring barley and wild oat Avena fatua L. or common couch Agropyron repens (L.) Beauv. Proc. Eur. Weed Res. Soc. Symp. Status and Control of Grassweeds in Europe, p. 77-86.
Erviö, L.-R. 1971. The effect of intra-specific competition on the development of Chenopodium album L. Weed Res. 11: 124134.

Erviö, L.-R. 1972. Growth of weeds in cereal populations. J. Sci. Agric. Soc. Finl. 44: 19-28.

Gill, G.S., M.L. Poole et J.E. Holmes. 1987. Competition between wheat and brome grass in Western Australia. Aust. J. Exp. Agric. 27: 291-294.

Harrison, S.K. 1990. Interference and seed production by common lambsquarters (Chenopodium album) in soybeans (Glycine max). Weed Sci. 38: 113-118.

Isaac, R. A. et W.C. Johnson. 1976. Determination of total nitrogen in plant tissue, using a block digestor. J. Assoc. Off. Anal. Chem. 59: 98-100.

Kapoor, P. et P.S. Ramakrishnan. 1978. Mortal and plastic responses of pure and mixed populations of wheat and Chenopodium album L. at two levels of nutrition. Proc. Indian Acad. Sci. 87: 243-248.

Koscelny, J.A., T.F. Peeper, J.B. Soli et S.G. Solomon, Jr. 1990. Effect of wheat (Triticum aestivum) row spacing, seeding rate, and cultivar on yield loss from cheat (Bromus secalinus). Weed Technol. 4: 487 492.

Koscelny, J.A., T.F. Peeper, J.B. Soli et S.G. Solomon, Jr. 1991. Seeding date, seeding rate, and row spacing affect wheat (Triticum aestivum) and cheat (Bromus secalinus). Weed Technol. 5: 707-712.

Lachat Instruments. 1992. QuickChem method 13-107-06-2-D. Total Kjeldahl Nitrogen in Soil/Plant. Lachat Instruments, Milwaukee, USA, $10 \mathrm{pp}$.

Lapointe, A.-M., J.-M. Deschênes, P. Gervais et C. Lemieux. 1984. Interférence du chénopode blanc (Chenopodium album), chez l'avoine et la luzerne : seuils de nuisibilité. Can. J. Bot. 62: 2594-2599.

Lemieux, C., A. Larouche, A. Légère, J.-M. Deschênes et R. Rioux. 1988. Inventaire des mauvaises herbes au Québec; Cultures céréalières. Bulletin d'extension 2. Agriculture Canada, Sainte-Foy, Québec, 84 pp.

Malicki, L. et C. Berbeciowa. 1986. Uptake of more important mineral components by common field weeds on loess soils. Acta Agrobot. 39: 129-141.

Mann, H.H. et T.W. Barnes. 1950. The competition between barley and certain weeds under controlled conditions. IV. Competition with Stellaria media. Ann. Appl. Biol. 37: 138-148.

Mclennan, B.R., R. Ashford et M.D. Devine. 1991. Cirsium arvense (L.) Scop. competition with winter wheat (Triticum aestivum L.). Weed Res. 31: 409-415. 
Morishita, D.W. et D.C. Thill. 1988. Wild oat (Avena fatua) and spring barley (Hordeum vulgare) growth and development in monoculture and mixed culture. Weed Sci. 36: 43-48.

Pageau, D. et G.F. Tremblay. 1995. Effet du chiendent sur l'orge ensemencée à différents écartements entre les rangs et doses de semis. Can. J. Plant Sci. 75: 613618.

Peterson, D.E. et J.D. Nalewaja. 1992. Green foxtail (Setaria viridis) competition with spring wheat (Triticum aestivum). Weed Technol. 6: 291-296.

Richards, J.E. 1993. Chemical characterization of plant tissue. Pages 115-139 in M.R. Carter (ed.), Soil sampling and methods of analysis. Lewis publ., USA.

SAS Institute inc. 1990. SAS/STAT user's guide. Version 6, $4^{\text {th }}$ Edition, Vol. 1 et 2. SAS Institute Inc., Cary, North Carolina. $638 \mathrm{pp}$.

Shahi, H.N. 1978. Competitive effects of Chenopodium album for soil moisture and nutrients in wheat. Integ. Pest Control 62: 14-16.

Sibuga, K.P. et J.D. Bandeen. 1980. Effects of green foxtail and lamb's-quarters interference in field corn. Can. J. Plant Sci. 60: 1419-1425.
Solie, J.B., S.G. Solomon Jr., K.P. Self, T.F. Peeper et J.A. Koscelny. 1991. Reduced row spacing for improved wheat yields in weed-free and weed-infested fields. Trans. ASAE 34: 1654-1660.

Szente, K., Z. Tuba, Z. Nagy et Z.S. Csintalan. 1993. Competition between Chenopodium album and Helianthus annuus as reflected in photosynthesis and transpiration. Photosynthetica 28: 465-472.

Vengris, J., M. Drake, W.G. Colby et J. Bart. 1955. Chemical composition of weeds and accompanying crop plants. Agron. J. 45: 213-218.

Welbank, P.J. 1963. A comparison of competitive effects of some common weed species. Ann. Appl. Biol. 51: 107-125.

Wicks, G.A., R.E. Ramsel, P.T. Nordquist, J.W. Schmidt et Challaiah. 1986. Impact of wheat cultivars on establishment and suppression of summer annual weeds. Agron. J. 78: 59-62.

Willey, R.W. et R. Holliday. 1971. Plant population, shading and thinning studies in wheat. J. Agric. Sci. 77: 453-461. 